Case

Series

Teoman Kilic, MD

Tayfun Sahin, MD

Ertan Ural, MD
Key words: Cardiac catheterization/instrumentation; heart valve prosthesis implantation/instrumentation/ methods; mitral valve/ pathology; mitral valve insufficiency/etiology/therapy; prosthesis implantation/ adverse effects/methods; septal occluder device; treatment outcome

From: Department of Cardiology (Drs. Kilic, Sahin, and Ural), Kocaeli University Medical Faculty; and Invasive Cardiology Research and Application Unit (Drs. Kilic and Ural), Kocaeli University, 41380 Kocaeli, Turkey

Address for reprints: Teoman Kilic, MD, Invasive Cardiology Research and Application Unit, Department of Cardiology, Umuttepe Campus, Kocaeli University, 41380 Kocaeli, Turkey

E-mail:

kilicteoman@yahoo.com

(C) 2014 by the Texas Heart ${ }^{\circledR}$ Institute, Houston

\section{Percutaneous Retrograde Transfemoral Closure of Mitral Paravalvular Leak}

\author{
in 3 Patients without Construction of an \\ Arteriovenous Wire Loop
}

Percutaneous closure of paravalvular leaks has emerged as an alternative to repeated surgeries. Different percutaneous techniques and various devices have been used, off-label, for paravalvular leak closure. For mitral leaks, antegrade transseptal, retrograde transfemoral, and retrograde transapical techniques have been developed. In the antegrade transseptal approach, an arteriovenous guidewire loop is often created to advance the delivery sheath. In retrograde transfemoral closure, the wire in the left atrium is usually snared after transseptal puncture, to pull it from the femoral vein. The delivery sheath and closure device will subsequently be deployed from the left atrium. Each of these procedures takes time, is costly, and increases the risk of complications.

We present the cases of 3 patients in whom we closed mitral paravalvular leaks by means of a retrograde transfemoral approach, with use of an AMPLATZER ${ }^{\text {TM }}$ Duct Occluder II device and without the construction of an arteriovenous wire loop. We think that this approach can be very useful in a specific group of patients-reducing costs, fluoroscopy times, and complications related to transseptal puncture and construction of an arteriovenous wire loop. In our institution, this reported technique is routinely used for mitral paravalvular leak closure. (Tex Heart Inst J 2014;41(2):170-3)

\footnotetext{
$\mathbf{P}$
} aravalvular leak (PVL), an important sequela in the surgical replacement of aortic and mitral valves, occurs in $8 \%$ to $18 \%$ of patients after valvular surgery. ${ }^{1}$ Depending on the size of the leak, patients can be asymptomatic or can present with congestive heart failure, hemolytic anemia, or both. In addition, severe PVL increases the risk of infective endocarditis and arrhythmia. ${ }^{2-4}$ In symptomatic patients, surgery is the gold standard of treatment. Reoperation is associated with an increased risk of morbidity and death. Recurrent leaks after reoperation are caused by underlying tissue friability, inflammation, or calcification. ${ }^{2,3}$

Percutaneous transcatheter closure of PVL has emerged as an alternative to repeat surgery. ${ }^{1-4}$ Different techniques and devices have been used off-label. ${ }^{4,5}$ We present the cases of 3 patients whose mitral paravalvular leaks were closed with use of an AmPLATZER $^{\text {TM }}$ Duct Occluder II (ADO II) (St. Jude Medical, Inc.; St. Paul, Minn) by means of a retrograde transfemoral approach and without the construction of an arteriovenous wire loop.

\section{Case Summaries}

Patient 1. In 2007, a 77-year-old man was diagnosed with chronic obstructive pulmonary disease and severe mitral regurgitation caused by mitral valve prolapse, flail leaflet, and chordae rupture. That year, he underwent mitral valve replacement (MVR) with implantation of a 29-mm bileaflet St. Jude mechanical valve. Upon admission to our clinic in March 2012, he reported dyspnea, fatigue, and weight loss. Transthoracic echocardiograms revealed moderate-to-severe mitral regurgitation. Two-dimensional transesophageal echocardiograms (2D TEE) showed substantial PVL in the posteromedial region (Fig. 1A). The patient's EuroSCORE II was 11.02\%, and he was at high risk for repeat surgery. He declined reoperation and agreed to undergo percutaneous closure of the defect. 
Patient 2. In 2011, a 68-year-old woman underwent MVR with implantation of a 27-mm bileaflet St. Jude mechanical valve. Two months later, prosthetic valve endocarditis developed, and MVR with an implanted bioprosthesis was performed. In June 2012, we found substantial PVL in the lateral region (Fig. 1B). The patient's EuroSCORE II was $12.2 \%$. Because of her risk status and history of repeated MVR, we decided to perform percutaneous PVL closure.

Patient 3. In 2005, a 71-year-old man with chronic kidney disease underwent MVR with implantation
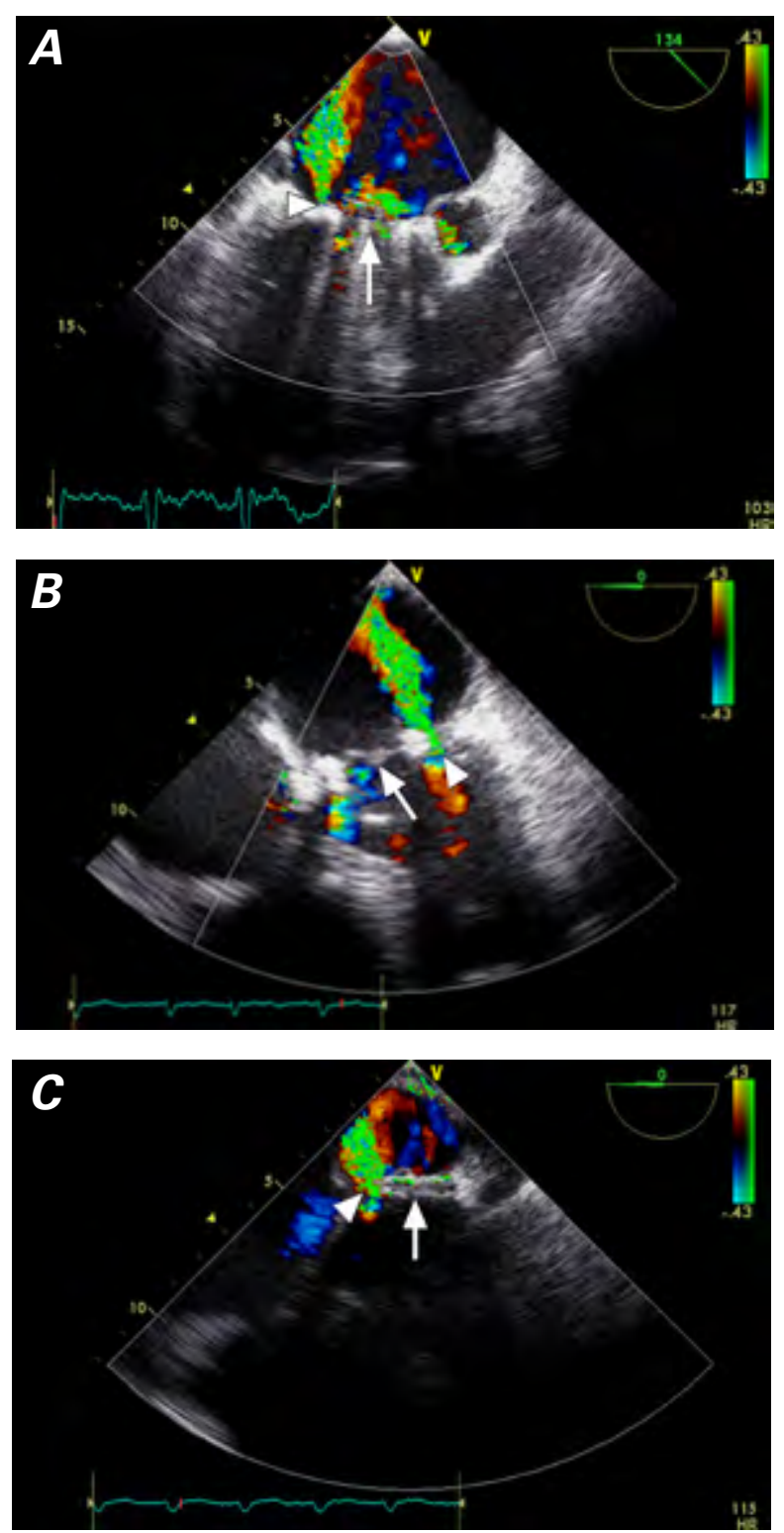

Fig. 1 Transesophageal echocardiograms show severe mitral paravalvular regurgitant jets (arrowheads) in A) Patient 1's posteromedial region (mechanical mitral valve and its acoustic shadowing, arrow), B) Patient 2's lateral region (bioprosthetic mitral valve, arrow), and C) Patient 3's posteromedial region (bioprosthetic mitral valve, arrow). of a $27-\mathrm{mm}$ bileaflet St. Jude mechanical valve. Three months later, because of infective endocarditis and septic cranial embolism, the first valve was replaced with a 27-mm Edwards porcine bioprosthetic valve (Edwards Lifesciences Corporation; Irvine, Calif). In 2008, he underwent DDDR pacemaker implantation. In June 2012, we found substantial PVL in the posteromedial region (Fig. 1C). The patient's EuroSCORE II was $12.41 \%$. He was at high risk for surgical MVR, so we decided to perform percutaneous PVL closure.

\section{Interventional Technique}

Written consent was obtained, and the PVL closure procedures were performed with the patients under deep sedation without endotracheal intubation. Fluoroscopic views of the femoral head were rapidly obtained so that we could see the proximity of the common femoral artery path. The skin entry site was marked with a clamp, and a high femoral puncture was performed. A 7F sheath was inserted into the left common femoral artery. We used a 6F diagnostic Amplatz left one (AL1) catheter to cross the PVL in each patient. The optimal projection to see and cross the PVL was a right anterior oblique (RAO) cranial view $\left(40^{\circ} \mathrm{RAO}\right.$ with $20^{\circ}$ cranial angulation). After introducing the catheter to the apex of the left ventricle (LV), we used push-and-pull maneuvers to cross the defects. We pushed the catheter to the LV apex, and in patients 1 and 3, we pulled the catheter tip back 2 to $3 \mathrm{~cm}$ in a clockwise rotation as the paravalvular defects were identified in the posteromedial regions. This maneuver enabled us to sweep the catheter tip in a posteromedial direction and cross the defect easily. In patient 2, we pushed the AL1 catheter to the LV apex and slightly pulled back the catheter tip in a counterclockwise rotation, which caused the catheter tip to sweep laterally and cross the PVL. After the PVL was crossed, a straight-tipped Terumo Glidewire ${ }^{\circledR}$ guidewire (Terumo Medical Corporation; Somerset, $\mathrm{NJ}$ ) was inserted into the catheter and advanced in the left atrium over the Terumo guidewire. The guidewire was then exchanged for an Amplatz extra-stiff guidewire (Cook Medical, Inc.; Bloomington, Ind).

\section{Device Deployment}

Patient 1. In Patient 1, the 6F AL1 diagnostic catheter was replaced with a 7F AL1 guiding catheter, which also served for device delivery. The stiff guidewire was withdrawn, and an ADO II with a 5-mm waist size and $12-\mathrm{mm}$ disc diameter was advanced through the catheter. Proper positioning was attained by means of 2D TEE guidance, and the occluder was deployed (Fig. 2A). The paravalvular regurgitation decreased immediately after the procedure. The total procedural time was $75 \mathrm{~min}$ and the fluoroscopic time was $21 \mathrm{~min}$. During follow-up evaluation, the patient reported less dyspnea and fatigue than before. A TEE 2 months after the pro- 

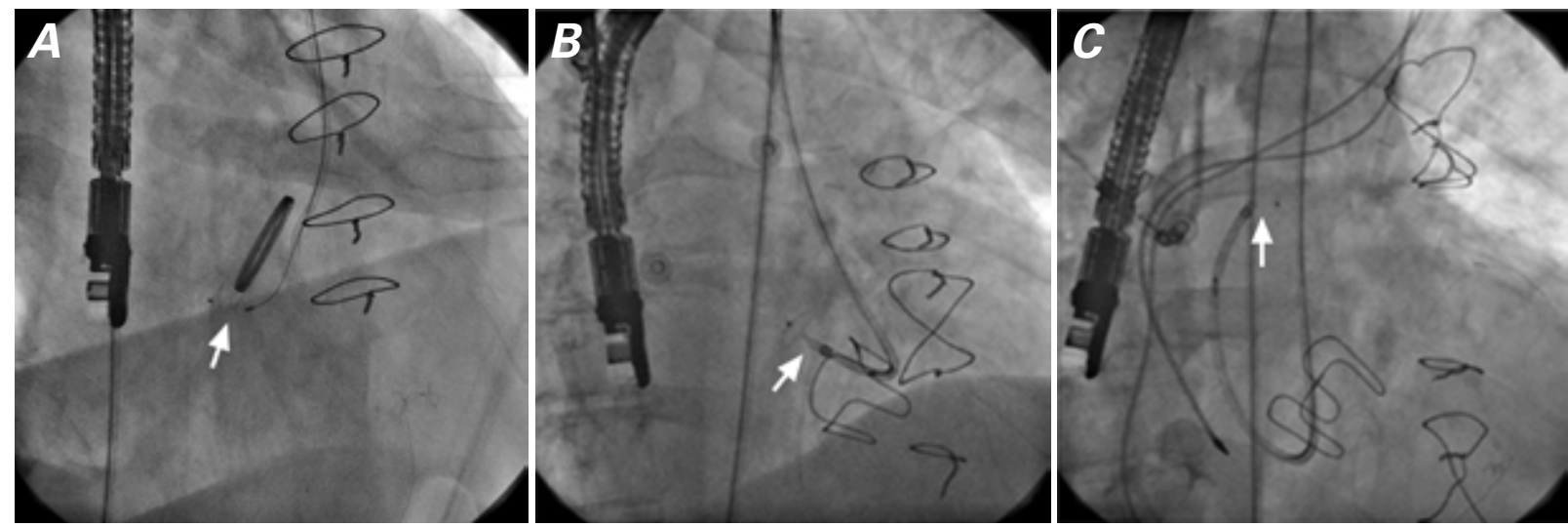

Fig. 2 Fluoroscopic images (right anterior oblique 40\%cranial $20^{\circ}$ views). A) Patient 1. Shown are the AMPLATZERTM Duct Occluder (ADO) II (arrow), the delivery cable, and the TF Amplatz 1 guiding and delivery catheter. The ADO II devices (arrows) and the AMPLATZER ${ }^{\text {TM }}$ Torqvue ${ }^{T M}$ delivery sheaths are shown in B) Patient 2 and $\boldsymbol{C}$ ) Patient 3.
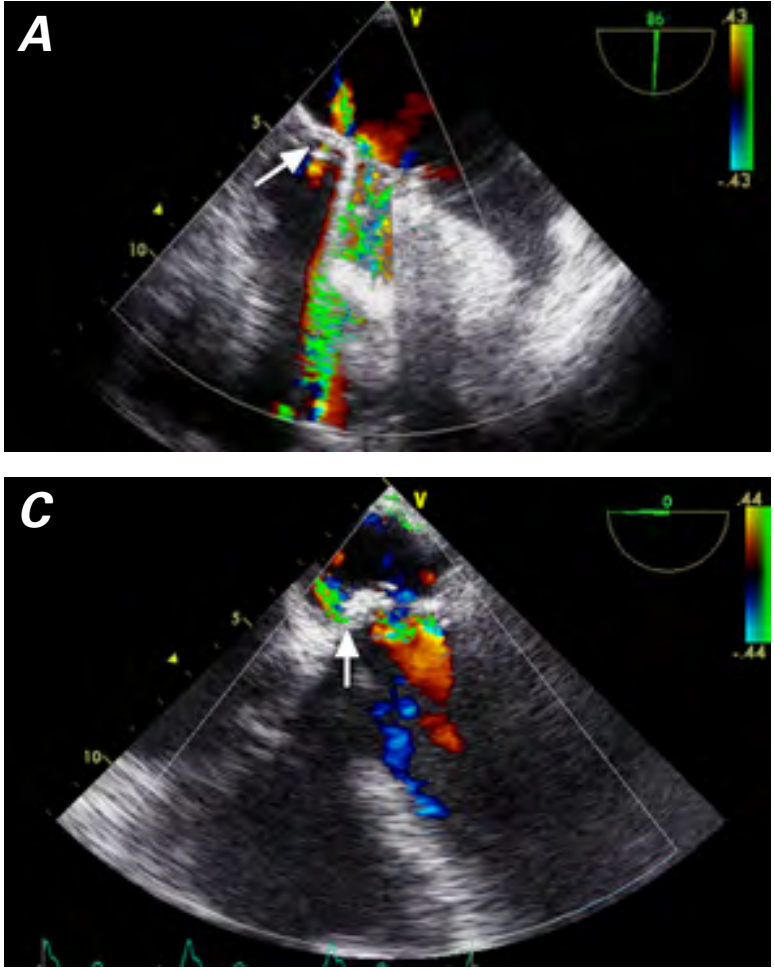

cedure showed mild regurgitation across the device (Fig. 3A).

Patient 2. In Patient 2, we used a 5F, $120-\mathrm{cm}-$ long AMPLATZER ${ }^{\mathrm{TM}}$ Torqvue ${ }^{\mathrm{TM}}$ delivery sheath (St. Jude Medical) as the delivery catheter (Fig. 2B), instead of a shorter 7F AL1 guiding catheter. An ADO II (waist size, $5 \mathrm{~mm}$; disc diameter, $12 \mathrm{~mm}$ ) was deployed under 2D TEE guidance (Fig. 2B). The PVL completely disappeared after the procedure (Fig. 3B). The procedural time was $70 \mathrm{~min}$ and the fluoroscopic time was $18 \mathrm{~min}$. The patient was discharged from the hospital 2 days after the intervention, in stable condition.

Patient 3. In Patient 3, a 5F, 120-cm-long AMPLATZER Torque delivery sheath was used to deploy an ADO

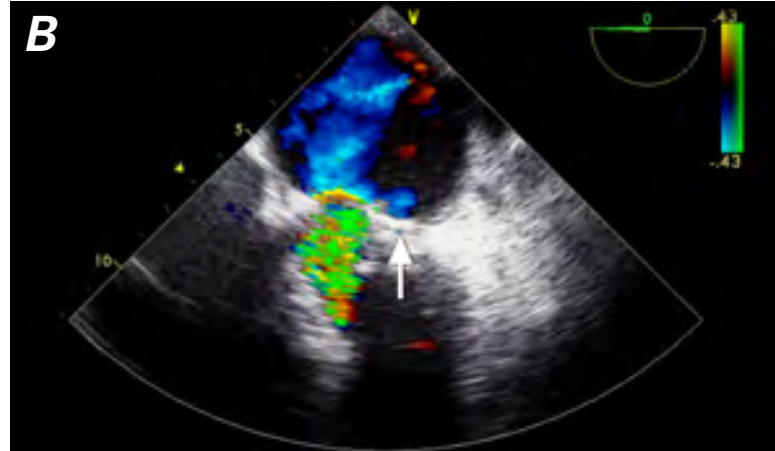

Fig. 3 Postprocedural transesophageal echocardiograms show the AMPLATZER ${ }^{\text {TM }}$ Duct Occluder I/ (arrows) and $\boldsymbol{A}$ ) decreased mitral paravalvular leakage in Patient 1, B) the complete disappearance of paravalvular leakage in Patient 2, and $\mathbf{C}$ ) markedly decreased leakage in Patient 3.

II (waist size, $6 \mathrm{~mm}$; disc diameter, $12 \mathrm{~mm}$ ) under 2D TEE guidance (Fig. 2C). Subsequently, the PVL markedly decreased. The procedural time was $50 \mathrm{~min}$ and the fluoroscopic time was $17 \mathrm{~min}$. The patient was discharged from the hospital one week after the intervention, in stable condition. A TEE 2 months after the procedure showed mild regurgitation across the device (Fig. 3C).

\section{Discussion}

Different techniques have been adopted for aortic and mitral PVL closures. For aortic PVL, retrograde transfemoral cannulation is preferred, and antegrade 
transapical access is generally recommended as a backup option, especially in patients in whom retrograde attempts have failed. ${ }^{3-5}$ For mitral PVL, the most frequently applied procedures are antegrade cannulation of the defect through a transseptal puncture, retrograde cannulation from the LV through the aortic valve, and retrograde cannulation via transapical access. ${ }^{6}$ In the antegrade transseptal and retrograde transfemoral approaches, many operators prefer to create an arteriovenous wire loop. However, each of these procedures takes time, increases cost, and increases the risk of complications. ${ }^{6,7}$ In addition, studies indicate that mitral PVL closure is more complex than aortic PVL closure. ${ }^{6,7}$

It is possible to achieve retrograde transfemoral closure of mitral PVL without creating a wire loop. Kursaklioglu and colleagues ${ }^{7}$ published the first relevant report, a single case. However, the technique has apparently not been evaluated further in any group of patients. We modified the technique and applied it in our 3 patients, one of whom had a mechanical prosthetic valve and the others, bioprosthetic valves.

In all 3 patients, we used a high femoral puncture technique, because the distance between the groin and PVL is usually too long for a standard diagnostic catheter in retrograde approach. Whereas Kursaklioglu and colleagues used left anterior oblique projections, we preferred $\mathrm{RAO} 40^{\circ}$ with cranial $20^{\circ}$ projections, which enabled a good view of the mitral annulus and facilitated crossing the PVL and deploying the closure device. To cross the PVL, we selected the diagnostic AL1 catheter, because its retroflex shape was suitable for our push-and-pull maneuvers. In patients 2 and 3 , we used the long, 5F AMPLATZER Torqvue delivery sheath instead of a short $7 \mathrm{~F}$ guiding catheter, because the sheath provided better support, enabled better fluoroscopic visibility, and had more of a slick feeling during device deployment. Although high femoral puncture helped us to cross the PVL easily with these catheters, this technique would benefit from specially designed, especially long atraumatic catheters for crossing defects and deploying closure devices.

We used the ADO II for retrograde transfemoral mitral PVL closure, because we could open its distal disc in a low-pressure chamber (the left atrium), and because the device is available in small waist sizes and small disc diameters designed to avoid overlap with mechanical valve leaflets. ${ }^{7}$ However, other devices with double discs, such as the AMPLATZER Vascular Plug III (St. Jude Medical) or muscular ventricular septal defect occluders, can also be used in this technique. One limitation of the procedure as described is our inability to perform PVL closure under 3-dimensional (3D) TEE guidance. Our main objective here is to describe the versatility of this percutaneous technique while pointing out that it can be combined with 3D TEE or other imaging tools.
We think that the percutaneous transfemoral retrograde closure of mitral PVL without creating an arteriovenous wire loop can be very useful in high-risk patients. The technique can reduce costs, fluoroscopy times, and complications, particularly those related to transseptal puncture and the construction of an arteriovenous wire loop. This approach has become standard for mitral PVL closure at our institution.

\section{References}

1. Sorajja P, Cabalka AK, Hagler DJ, Rihal CS. Long-term follow-up of percutaneous repair of paravalvular prosthetic regurgitation. J Am Coll Cardiol 2011;58(21):2218-24.

2. Rihal CS, Sorajja P, Booker JD, Hagler DJ, Cabalka AK. Principles of percutaneous paravalvular leak closure. JACC Cardiovasc Interv 2012;5(2):121-30.

3. Buellesfeld L, Meier B. Treatment of paravalvular leaks through interventional techniques. Multimedia Man Cardiothorac Surg. Available from: http://mmcts.oxfordjournals. org/content/2011/0924/mmcts.2010.004895.full.pdf + html [cited 2013 Nov 11].

4. Kim MS, Casserly IP, Garcia JA, Klein AJ, Salcedo EE, Carroll JD. Percutaneous transcatheter closure of prosthetic mitral paravalvular leaks: are we there yet? JACC Cardiovasc Interv 2009;2(2):81-90.

5. Pate GE, Thompson CR, Munt BI, Webb JG. Techniques for percutaneous closure of prosthetic paravalvular leaks. Catheter Cardiovasc Interv 2006;67(1):158-66.

6. Noble $S$, Basmadjian A, Ibrahim R. Transcatheter prosthetic paravalvular leak closure. Cardiovasc Med 2012;15(9):24552. Available from: http://www.cardiovascmed.ch/docs/ 2012/2012-09/2012-09-053.PDF [cited 2013 Nov 11].

7. Kursaklioglu H, Barcin C, Iyisoy A, Baysan O, Celik T, Kose S. Percutaneous closure of mitral paravalvular leak via retrograde approach with use of the Amplatzer duct occluder II and without a wire loop. Tex Heart Inst J 2010;37(4):461-4. 